\title{
Counter-Argument Self-Efficacy Predicts Choice of Belief-Defense Strategies
}

Matthew H. Goldberg ${ }^{1, *}$, Cheryl L. Carmichael ${ }^{2,3}$, and Curtis D. Hardin ${ }^{2,3}$

\author{
${ }^{1}$ Yale University \\ ${ }^{2}$ Graduate Center, City University of New York \\ ${ }^{3}$ Brooklyn College, City University of New York
}

This article has been accepted for publication at the European Journal of Social Psychology. Please cite as: Goldberg, M. H., Carmichael, C. L., \& Hardin, C. D. (in press). CounterArgument Self-Efficacy Predicts Choice of Belief-Defense Strategies. European Journal of Social Psychology.

Data Archiving and Sharing: All data are available at https://osf.io/ks5hf/.

Acknowledgments: Thank you to Joe Siev for assistance with writing the arguments for the experiments and for contributing useful points in our early discussions on argument strength. Thank you to Chris Gettings for inspiring many of these research questions in early discussions about cognitive dissonance.

${ }^{*}$ Correspondence: Matthew H. Goldberg; Yale University, 205 Prospect St., New Haven, CT, 06511; E-mail: matthew.goldberg@yale.edu 


\begin{abstract}
Research has identified many strategies people use to defend against belief-inconsistent information. However, little research has identified factors that predict which defense strategy people will use when more than one is available. Two experiments tested whether people choose to counter-argue belief-inconsistent information because they believe arguing will be successful, but resort to weaker defense strategies because they believe arguing will be unsuccessful. Exposure to strong versus weak belief-inconsistent information caused a decrease in counterarguing and an increase in ignoring (Experiment 1) or claiming a belief to be a matter of opinion (untestable) rather than a matter of fact (testable; Experiment 2). Consistent with self-efficacy theory, expectations of successful counter-arguing was the mechanism responsible for both effects. When people feel less capable of successfully counter-arguing because the information is too difficult to refute, they resort to epistemically weaker defense strategies in order to preserve their belief. Keywords: self-efficacy, belief defense, dissonance, attitudes
\end{abstract}




\section{Counter-Argument Self-Efficacy Predicts Choice of Belief-Defense Strategies}

People are motivated to defend deeply held beliefs when they are threatened, and do so using a wide variety of strategies (Kruglanski, 1996; Kunda, 1990). For example, people spend more effort criticizing belief-threating information (Edwards \& Smith, 1996) and apply stricter rules for accepting it (Ditto \& Lopez, 1992) compared to belief-consistent information.

Moreover, people ignore belief-threatening information by selectively seeking out information that confirms their beliefs, particularly when threatened (Eagly \& Chaiken, 1993; Hart, Albarracín, Eagly, Brechan, Lindberg, \& Merrill, 2009). Remarkably, people sometimes frame beliefs in terms that make them impossible to prove true, at least if it makes them impossible to prove false (Freisen, Campbell, \& Kay, 2014). For example, framing one's opposition on the death penalty as a matter of moral or personal opinion (e.g., "It is wrong to kill another person") rather than empirical fact (e.g., "It does not deter crime") protects the belief from refutation by making it empirically untestable (or "unfalsifiable;" see Freisen et al., 2014), but also means that one cannot defend the belief on the merits.

Although research has identified several belief-defense strategies, each strategy has been investigated in isolation, one at a time. Perhaps because of this, little if any research has identified a framework for predicting when and why people choose one defense strategy over another. Predicting people's choice of defense strategy is crucial because it can inform applied research into messaging campaigns on important social, political, and public health issues. For example, defensiveness against an unfavorable medical test has been shown to cause people to doubt the accuracy of the medical test and create stronger decision rules for accepting its conclusions (Ditto \& Lopez, 1992). If researchers developed a framework for predicting people's 
methods of defensive responding, messaging can be catered to addressing the defensive methods that people are most likely to use.

\section{Self Efficacy Theory and Belief Defense}

Given that people typically have multiple belief-defense strategies available in a given moment, which do they choose and why? No theoretical framework precisely predicts the answer to this question, but several are suggestive. Here we argue that one answer lies in people's expectations about their ability to effectively defend against the belief threat. One theory that deals most directly with people's beliefs about their ability to achieve a goal (in this case, beliefdefense) is self-efficacy theory (SET; Bandura, 1977).

Self-efficacy theory defines an efficacy expectation as “...the conviction that one can successfully execute the behavior required to produce the outcomes" (Bandura, 1977, p. 193). This theory postulates that when people have high self-efficacy, they are more likely to engage in active efforts to achieve a goal, even in the face of obstacles. For example, SET has been shown to predict performance in school (Bandura, Barbaranelli, Caprara, \& Pastorelli, 1996) and at work (Stajkovic \& Luthans, 1998).

Although SET accounts for a wide range of phenomena, it makes no specific predictions about the manner in which people will go about defending their beliefs, such as whether counterarguing is a more desirable form of belief-defense than ignoring. For example, one might perceive themselves as fully capable (i.e., high self-efficacy) of successfully counter-arguing information that contradicts their important beliefs, but there might be no incentive to do so if ignoring the information just as effectively allows a person to successfully defend a belief.

A large body of evidence suggests that people are largely cognitive misers, disinclined to expend energy, and ready to opt for the least demanding means of attaining a goal. For example, 
dual process models show that people often rely on easily processed peripheral cues (e.g., source credibility, number of arguments) when evaluating the quality of arguments instead of spending more energy evaluating the content of the arguments (Chaiken, 1980; Petty \& Cacioppo, 1986). Indeed, people often use low-demand methods of belief defense such as avoiding beliefinconsistent information instead of engaging with it (Hart et al., 2009).

However, as is readily apparent in any sports bar or internet discussion board, people often argue and counter-argue, despite the cognitive effort and social risk. People do this when they could save effort by, for example, ignoring belief-inconsistent information or asserting their beliefs are a matter of opinion and therefore not subject to empirical criticism. A likely reason for this effortful behavior is that counter-arguing may be more instrumental to the goal of successful belief-defense than other methods that do not refute belief-threats (e.g., ignoring, untestability; see Ahluwalia, 2000; Stone, Wiegand, Cooper, \& Aronson, 1997). Supporting this idea, multiple studies show that counter-arguing strong information increases attitude certainty (Tormala \& Petty 2002; 2004).

Self-efficacy theory also suggests that counter-arguing may be worth the effort if it better serves the goal of belief-defense than other methods. Bandura (1977) theorized:

...there are many things that people can do with certainty of success that they do not perform because they have no incentives to do so. Given the appropriate skills and adequate incentives, however, efficacy expectations are a major determinant of people's choice of activities... (Bandura, 1977; p. 194)

If successful counter-arguing provides a more effective (i.e., higher incentive) belief-defense than ignoring, for example, then expectations of efficacy should predict decisions to counterargue over other methods of belief-defense. 
This extension of self-efficacy theory makes an important contribution to the literature on belief-defense because it clarifies how people approach belief-defense when there are multiple means to achieving it. Further, a primary contribution is gaining a better understanding of whether people will resort to other methods of belief-defense when they believe they will be unsuccessful at their preferred strategy. In the current experiments, we demonstrate that people will employ the strongest possible method of belief defense they believe will be successful instead of opting for the easiest defense (e.g., ignoring or engaging only when the threat is weak; Lowin, 1967). In other words, feeling efficacious about the ability to successfully counterargue will be associated with people's decisions to directly refute belief-inconsistent information. Conversely, low self-efficacy will be associated with people's decisions to enlist methods that do not refute the belief-inconsistent information (e.g., ignoring, untestability), but preserve the belief in a less direct manner.

\section{Dual-Process and Dissonance in Belief Defense}

Dual process theories identify two routes to persuasion, and more specifically, beliefdefense (e.g., Chaiken, 1980; Kruglanski et al., 2012; Petty \& Cacioppo, 1986). For example, the elaboration likelihood model (Petty \& Cacioppo, 1986) states that people may be persuaded by the central or peripheral route. Central route persuasion occurs via argument content whereas peripheral route persuasion occurs via content unrelated to the argument, such as the length or number of arguments.

Whether people take the central or peripheral route depends on people's motivation and ability. That is, people will only invest energy scrutinizing argument content if they are both motivated and able to do so. For example, political partisans might be highly motivated to defend their favorite candidate's tax policy on its merits but are limited by their lack of 
knowledge. Lacking knowledge would constrain their choice of belief defense strategies and would therefore decrease the likelihood of defending their beliefs through the central route (e.g., counter-arguing), and increase the likelihood that other, peripheral methods will be used (e.g., ignoring, untestability).

Cognitive energetics theory (CET; Kruglanski, Bélanger, Chen, Köpetz, Pierro, \& Mannetti, 2012) makes similar predictions. The theory assumes that attainment of a cognitive goal (e.g., successful belief defense) is guided by a driving force (the maximal amount of energy that a person is willing to invest in attaining a cognitive goal) and a restraining force (factors inhibiting goal pursuit). Driving forces include the importance of attaining the cognitive goal, and the amount of available mental resources, whereas task difficulty is a restraining force. Reducing the quality of belief-inconsistent arguments should decrease the restraining force, and simultaneously increase perceived ability to counter-argue (the driving force). CET states that when people have a high driving force, such as high motivation to defend an important belief, they choose the most effective way of attaining their goal even if that means investing more energy.

Although dual-process theories and cognitive energetics theory suggest that, with sufficient motivation, people are willing to invest substantial energy in defending personally important beliefs, it is still unknown whether people would prefer to engage in counter-arguing if other, perhaps less effortful, methods were available. We turn to the literature on cognitive dissonance theory to help fill this knowledge gap and identify direct evidence that speaks to this research question.

Cognitive dissonance is the psychological discomfort people have when they hold two conflicting attitudes, beliefs, or behaviors (Festinger, 1957). People are motivated to reduce this 
discomfort, and can do so by changing one or more of the inconsistent elements. For example, if a political partisan is scrolling through her Facebook feed and sees arguments against her beliefs on gun control, she could attempt to refute the belief-inconsistent information, criticize the source of the message, scroll by and ignore it, or choose from an array of other responses in attempts to reduce the psychological discomfort caused by the belief-inconsistent information.

Research shows that some methods of dissonance reduction are preferred to others. For example, direct methods of resolving dissonance (e.g., counter-arguing) are sometimes preferred to indirect methods (e.g., ignoring, untestability; Stone et. al., 1997). This is supported by Stone et al.'s (1997) research which used the hypocrisy paradigm to induce cognitive dissonance, and the motivation to reduce it. They asked participants to advocate for the use of condoms and then reflect on times they failed to use condoms. Participants were given a choice between a direct or indirect method of reducing dissonance. The direct method addressed the inconsistency; participants were given a chance to buy condoms. The indirect method did not address the inconsistency but allowed participants to reduce the negative state of dissonance by restoring self-integrity in a different domain (donating to the homeless). In two experiments, participants preferred the direct method of dissonance reduction, even when the indirect method was readily available, and required relatively less effort. People did, however, make ample use of the indirect strategy when it was the only one available. Viewing this work through the lens of self-efficacy theory, people were willing to expend greater effort to more effectively reduce dissonance by directly addressing the inconsistency.

Similarly, Simon, Greenberg, and Brehm (1995) found that people are likely to use trivialization as a mode of dissonance reduction instead of the more direct approach of attitude change, but only when they made the participants' initial attitude salient. For example, if a 
person has advocated for eating healthy foods, and then behaves inconsistently by eating junk food, they can reduce dissonance by changing their attitudes to be more in line with the current behavior (e.g., junk food is good) or trivialize their eating of junk food by asserting it was only this one time. Reminding people of their pre-existing advocacy for healthy eating makes trivialization a more desirable mode of dissonance reduction because it is salient to the person that they find healthy eating to be important. In terms of self-efficacy, making participants' original attitude salient likely made reducing dissonance via attitude change less effective and therefore trivialization became more desirable. Likewise, Voisin, Stone, and Becker (2013) found that people preferred trivialization and act-rationalization over attitude change only when changing one's attitude violated an important norm. The norm constrained participants' available dissonance reduction strategies by making attitude change less efficacious at reducing dissonance, thereby leading them to prefer trivialization and act-rationalization. Hence, one might be tempted to shift one's attitude on junk food to be more positive in order to justify having eaten some. But if doing so violates important norms in their social group (e.g., junk food is bad), then other methods of dissonance reduction would likely be more effective.

Together this research demonstrates that situational factors sometimes constrain people's dissonance reduction strategies to just one option, leading people to use whichever strategy is available. When there are multiple ways to reduce dissonance, however, people prefer methods that are most effective (i.e., directly addresses the inconsistency; Stone et al., 1997). The same logic may apply to the self-efficacy of belief-defense: people will, if possible, directly refute threats to their beliefs but when strong belief-inconsistent information lowers self-efficacy and makes people believe they are unable to refute the information itself, they will, in turn, resort to other available strategies such as ignoring or untestability. 


\section{Direct Evidence on Belief-Defense}

Belief-defense differs considerably from dissonance resulting from hypocrisy or writing counter-attitudinal essays. Most importantly, belief-defense is a response to an epistemic threat, eliciting a desire to defend what is believed to be true. Although no studies have directly examined choice of belief-defense strategies, evidence suggests that people may go to great lengths to respond to epistemic threats if their efforts are likely to succeed.

Despite the strong preference for information that confirms existing beliefs (Hart et al., 2009), people seek out belief-inconsistent information when it is weak and easy to refute, but not when it is strong and difficult to refute (Lowin, 1967). However, those studies did not include measures of people's perceived ability to successfully counter-argue the messages they received, thus it is not clear whether self-efficacy played a role in people's decisions.

In another line of research, Albarracín and Mitchell (2004) found that high confidence in the ability to defend one's beliefs (i.e., high efficacy) reduced people's bias in favor of beliefconsistent information; that is, they were more likely than people with low efficacy to engage with belief-inconsistent information. However, their research did not manipulate argument strength, so it is unclear whether high-efficacy participants would avoid strong but not weak belief-inconsistent information. Consistent with self-efficacy theory, both lines of research suggest that decisions to engage with strong belief-inconsistent information should be driven by the belief that one can successfully refute it.

A real-world example is evident in an analysis of people's attitudes when then-President Bill Clinton was under investigation for the Whitewater Scandal (Ahluwalia, 2000). Initially, Clinton supporters questioned the validity of the negative information against Clinton by attacking evidence of his dishonesty. As more information emerged, and it became harder for 
Clinton supporters to refute the president's dishonesty, they reduced the impact of the beliefinconsistent information by minimizing the role the trait honesty played in their support for Clinton and emphasizing other factors unrelated to honesty, such as trait intelligence. These findings suggest that people counter-argue the information when it is weak because they have high efficacy for doing so successfully. But when it is strong they resort to strategies that do not require a refutation of the information - including strategies that preclude one's ability to prove one's beliefs true- because the strong information led them to have low self-efficacy for counter-arguing.

Consider experimental evidence showing that people defended their beliefs by claiming that their belief was not empirically testable (Freisen et al., 2014). Reading belief-supporting information caused people to rate their reasons for their position as more "matter of fact" (i.e., testable) whereas reading belief-opposing information caused people to rate their reasons as more "matter of opinion" (i.e., untestable). By framing their beliefs as more "matter of opinion," people defended their beliefs from challenges by making them less subject to empirical disproof, but also rendered their belief impossible to prove true. Using this defense strategy is no small concession; to avoid being wrong people relinquish the ability to argue that their beliefs are correct. Thus, this really should be a strategy of last resort, perhaps to be used only when counter-arguing appears futile.

Similarly, ignoring belief-inconsistent information does nothing to validate beliefs. Because validating important beliefs is a fundamental motivation (Hart et al., 2009), defense strategies that relinquish refutation, such as ignoring it or claiming that it is untestable, should be less preferable because they forgo the opportunity to validate the belief. Even so, though ignoring and untestability are epistemically weak, they may minimize the impact of belief-threat 
and allow the belief to be retained when one is unable to successfully counter-argue. Thus, strong belief-discrepant arguments should lead people to believe they cannot successfully counter-argue (i.e., low efficacy), which, in turn, should be associated with the use of an epistemically weaker defense strategy such as ignoring or untestability.

\section{Current Research}

Our use of self-efficacy theory unifies and extends previous research in two ways. First, in our research participants are given a choice between methods of belief defense rather than giving participants a choice between belief-consistent and belief-inconsistent information. Second, and more importantly, whereas past research has examined the effects of either selfefficacy or task difficulty, the current experiments examine these factors simultaneously to understand belief-defense strategies.

In two experiments, the strength of belief-inconsistent information (weak vs. strong) was manipulated and expectations of efficacious counter-argument was measured to test whether it mediates the effect on choice of belief-defense (Experiment 1: counter-arguing vs. ignoring; Experiment 2: counter-arguing vs. untestability).

The purpose of the research reported here was to integrate seemingly disparate narratives into one theoretical framework. We hypothesize that people want to both effectively defend their beliefs and conserve cognitive resources. When a weak belief threat renders efficacy high, people will choose the most effective means to defend their belief. When a strong belief threat renders efficacy low, people will settle for weaker means to maintain their belief. Hence, the effect of argument strength on defense strategy will be mediated by expectations of counterargument efficacy. That is, compared to weak information, strong information will reduce people's expectations of effective counter-arguing, which, in turn, will be associated with 
increases in preferences for the weaker defense strategy (Experiment 1: ignoring; Experiment 2: untestability). Importantly, people will choose to counter-argue even strong information, as long as they believe they will be efficacious.

\section{Experiment 1}

\section{Method}

\section{Participants and Power Analysis}

We used the effect size from a similar experiment (Rios, Goldberg, \& Totton, 2018; Study 2) to estimate the effect size for this experiment. The primary finding had an odds ratio of 1.32, which requires 424 participants to detect with $80 \%$ power according to $G *$ Power (Faul, Erdfelder, Buchner, \& Lang, 2009).

Four hundred and twenty-eight people participated in a study of "Attitudes towards social issues" through Amazon's Mechanical Turk (MTurk). TurkPrime software (Litman, Robinson, \& Abberbock, 2016) was used to recruit participants from the U.S.A., with a minimum of $85 \%$ task approval, and a minimum 100 tasks completed. Ten participants were removed for failing an attention check. ${ }^{1}$ The final sample contained 418 participants $\left(M_{\text {age }}=37.22, S D_{\text {age }}=12.17\right.$; $48.1 \%$ male).

\section{Design}

Argument strength (Weak vs. Strong) was manipulated, expectations of effective counterarguing was measured as a potential mediator (four item composite), and choice of defense strategy was measured as the dependent variable (Counter-argue vs. Ignore) in a one-way, between-subjects design.

\section{Materials and Procedure}


All data and materials used in this research are available at https://osf.io/ks5hf/. ${ }^{2}$

Participants first read an overview of the study. To reduce participant attrition, we adapted the procedure from Zhou \& Fishbach (2016). Participants were given instructions and asked to type out "I will complete the full survey" and enter their MTurk identification number.

After completing a brief attention check task (Goldberg \& Carmichael, 2017), participants indicated their position on the death penalty and gun control (In Favor, Opposed), ${ }^{3}$ and the personal importance they assign to that issue ( 3 items per issue; $\alpha$ Gun Control $=.86, \alpha$ Death Penalty $=.89$ ). For personal importance of the issue of gun control, for example, participants answered, "How important is your attitude towards gun control laws to you?"; "To what extent does your attitude toward gun control laws reflect your core values?"; "How central is your attitude towards gun control laws to your sense of who you are?" $(1=$ Not at all, $9=$ Extremely $)$.

Then participants were randomly assigned to read either a strong or weak argument ${ }^{4}$ that contradicted their position on one of the two issues. ${ }^{5}$ For example, the weak argument in favor of gun control was:

The fact that guns are so widely accepted in American culture is harmful and federal law should be used to change this. With the laws as loose as they are now, people are too casual about gun ownership and don't take seriously enough the danger of these weapons. In a discussion with a friend of mine, he told me that he could buy a gun in his own state without even having to go through any training on gun laws or gun usage. We need to limit gun ownership in the U.S. so that we can do away with our gun obsession.

Whereas the strong argument in favor of gun control was:

Several promising strategies are available to legislators who want to reduce the number of annual gun fatalities in the U.S. The National Institutes of Health (NIH) estimates that 
a federal law requiring a waiting period of just one day would prevent at least one-third of annual gun suicides, saving more than 7,000 lives every year. Further, studies from the Centers for Disease Control (CDC) show that the majority of homicides are committed with legal firearms (about 68\%). The Bureau of Justice Statistics, the CDC, and independent researchers agree that making it more difficult to obtain guns will, even by conservative estimates, save thousands of lives per year.

Participants then completed a four-item scale designed to assess their expectations that they could successfully refute the contradictory message (Albarracín \& Mitchell, 2004). Using scales that ranged from 1 ("Extremely unlikely") to 9 ("Extremely likely"), participants rated how likely they were to "effectively counter-argue the argument above," "effectively refute the argument above," "identify problems in the argument above," and "know counter-arguments to the argument above," which were averaged to form a composite self-efficacy score $(\alpha=.95)$.

Participants then chose to either argue against the contradictory information or complete what they were told would be an unrelated task of equal time and difficulty. If participants chose to complete the counter-arguing task, they were instructed to "Please spend the next three minutes arguing against the message above." If participants chose to complete the unrelated task, they were instructed to "Please spend the next three minutes describing what political issue is most important to you and why." Finally, participants answered demographic questions.

\section{Results}

Before conducting our primary analyses, we first tested if participants were likely to be highly motivated to defend their beliefs. We used a one-sample t-test to test if participant ratings for personal importance of the issue were significantly above the scale's mid-point. Indeed, 
participant ratings of issue importance $(M=6.38, S D=1.86)$ were significantly higher than 5 on the 9-point scale, $t(417)=15.11, p<.001,95 \%$ CI $[1.20,1.56]$.

Did exposure to strong belief-inconsistent information cause participants to surrender an opportunity to counter-argue? A strong effect of argument strength on defense strategy choice indicates that the answer is yes. As revealed by a logistic regression analysis in which argument strength $(0=$ Weak, $1=$ Strong $)$ was entered in a model predicting task choice $(0=$ Counterargue, $1=$ Ignore) and shown in Table 1, people preferred to ignore the argument significantly more often when it was strong than when it was weak, $(b=.88, S E=.23), z(416)=3.89, p$ $<.001,95 \% \mathrm{CI}[.44,1.32]$. That is, participants had 2.41 times the odds of choosing to ignore than counter-argue when they received a strong argument than a weak argument, $\mathrm{OR}=2.41$, $95 \%$ CI $[1.55,3.74]$.

Table 1.

Percentage of participants choosing each defense strategy as a function of condition

\begin{tabular}{ccc} 
& Weak condition & Strong condition \\
\cline { 2 - 3 } Defense Strategy & \multicolumn{2}{c}{ Percentage of participants } \\
\hline Counter-argue & .38 & .20 \\
Ignore & .62 & .80 \\
\hline
\end{tabular}

More importantly, was choice of defense strategy explained by expectations of effective counter-arguing? Yes. A mediation analysis using the PROCESS macro in SPSS (Model 4; Hayes, 2013) confirmed the effect of argument strength on task choice was mediated by expectations of effective counter-arguing. As expected, compared to when participants received a weak argument, giving participants a strong argument significantly decreased participants' expectations that they could effectively counter-argue, $(b=-1.23, S E=.21), t(408)=-5.94, p$ $<.001,95 \% \mathrm{CI}[-1.64,-.82]$, which in turn was associated with preferences for ignoring instead of counter-arguing the message, $(b=-.50, S E=.25), z(407)=2.04, p<.001,95 \%$ CI $[-.64,-.35]$. 
(see Figure 1). Bootstrapping revealed that the indirect effect was significantly different from zero, $\left(b=.61, S E_{\text {boot }}=.14\right), 95 \% \mathrm{Cl}_{\text {boot }}[.38, .93]$. A test of moderated mediation revealed that the effect did not differ significantly by issue $\left(b=.14, S E_{\text {boot }}=.22\right), 95 \% \mathrm{CI}_{\text {boot }}[-.23, .63]$.

Figure 1. Mediation model testing the indirect effect of condition (weak vs. strong) on choice of defense strategy (counter-argue vs. ignore) through expectations of effective counter-arguing.
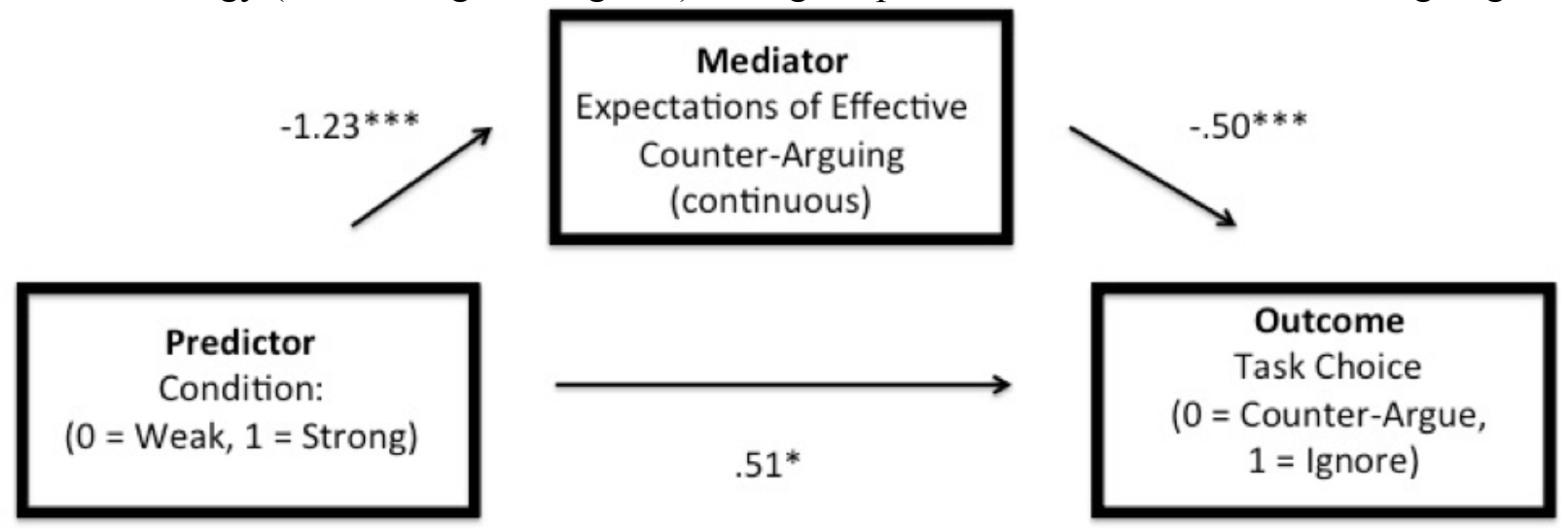

Note. $* p<.05, * * * p<.001$

Importantly, the path from the mediator (expectations of effective counter-arguing) to the outcome (choice of defense strategy) provides the test of whether participants who believed they could successfully counter-argue would choose to do so regardless of whether the argument was strong or weak. Each unit increase in expectations of effective counter-arguing was associated with $39 \%$ lower odds of choosing to ignore the message, $\mathrm{OR}=.61,95 \% \mathrm{CI}[.53, .70]$ (see Figure 1), even with argument strength in the model. Put another way, increased expectations that effective counter-arguing was associated with significantly greater odds of choosing to counterargue.

Finally, we tested if issue importance moderated the path from expectations of effective counter-arguing to choice of belief-defense (PROCESS Model 14). That is, was the indirect effect of condition stronger for people who found the issue to be of high personal importance? The test of moderated mediation was not significant, $\left(b=.03, S E_{\mathrm{boot}}=.04\right), 95 \% \mathrm{Cl}_{\mathrm{boot}}$ $[-.05, .11]$ 


\section{Discussion of Experiment 1 and Introduction to Experiment 2}

Results of Experiment 1 demonstrate that, compared to receiving strong beliefinconsistent information, weak information increased people's preference for counter-arguing against (versus ignoring) a challenge to their beliefs, and this was mediated by expectations that they will be successful. This clearly supports self-efficacy theory, which states that people who are motivated to reach a goal, such as belief-defense, will choose the best means of reaching that goal even if that means investing more energy. As opposed to other studies in which people may ignore information by not requesting it (Lowin, 1967) or by choosing belief-consistent information (Albarracín \& Mitchell, 2004), our results show that people's decision to counterargue was associated with their expectations that they would be successful. Although people preferred to ignore overall, the mediation model confirms that expectations of effective counterarguing was associated with the choice to counter-argue over ignoring the information. Crucially, expectations of effective counter-arguing (high self-efficacy) was significantly associated with choice of defense strategy even when controlling for argument quality, meaning that people will choose to counter-argue even strong arguments (high task difficulty), rather than doing so only when arguments are weak (Lowin, 1967).

Ignoring is just one of several ways to avoid counter-arguing, and is ambiguous with respect to just how unsure participants are of their beliefs in the face of a strongly contradictory information. For example, ignoring belief-threatening information does not necessarily mean that the person accepts that their belief has been refuted. An argument strong enough to persuade people that their belief may well not be able to stand on the merits might lead them to surrender the importance of testability, at least if a strategy remains that allows them to maintain that belief. If the motivation to maintain existing beliefs supersedes the motivation to be correct, then 
people may concede that they cannot win an argument but nevertheless maintain their belief by asserting that the issue is an untestable matter of opinion. The purpose of Experiment 2 was to test this possibility.

\section{Method}

\section{Participants and Power Analysis}

Effect sizes in closely related research on untestability (i.e., unfalsifiability) as a belief defense (Freisen et al., 2014; Study 4) were $\eta_{\mathrm{p}}^{2}=.052$ and .132 . To achieve the smaller effect of $\eta_{\mathrm{p}}^{2}=.052$ at $90 \%$ power, G*power (Faul et al., 2007) indicated 194 participants were needed. We raised our goal sample by $7 \%(+14$ participants $)$ to preserve statistical power after excluding participants for failing the attention check (see Goldberg \& Carmichael, 2017; Hauser \& Schwarz, 2016) making our goal sample size 208 participants. Additionally, the relatively large effect size in Experiment 1 and the use of one-tailed tests (as supported by the preregistered analysis plan) allowed for a smaller sample while maintaining excellent statistical power.

Two hundred and twenty-four people participated in this experiment through Amazon's Mechanical Turk $\left(M_{\text {age }}=35.36,{ }^{6} S D_{\text {age }}=11.53 ; 47.8 \%\right.$ male $)$. Like Experiment 1 , the TurkPrime software limited the study to participants in the U.S.A, with a task approval rate of at least $85 \%$, and to those who have completed at least 100 tasks (Litman et al., 2016). Eleven participants were excluded for failing the attention check, making our final sample $213{ }^{7}$

\section{Design}

The design is identical to Experiment 1 (one-way between-subjects). The only difference was the dependent variable. We manipulated argument strength (Weak vs. Strong) then measured our proposed mediator expectations of effective counter-arguing (four item 
composite). Choice of defense strategy was the dependent variable, where participants could choose either counter-arguing or untestability.

\section{Materials and Procedure}

All materials and preregistered hypotheses for this experiment are available at https://osf.io/ks5hf/. Participants read an overview of the experiment then completed questions that prevent participant attrition and detect mindless responding. Then, as in Experiment 1, participants indicated their position on gun control and the death penalty, rated the personal importance of each issue $\left(1=\right.$ Not at all, $9=$ Extremely; $\left.\alpha_{\text {Gun Control }}=.82, \alpha_{\text {Death Penalty }}=.88\right)$, and were randomly assigned to read either a weak or strong message criticizing their position on the randomly selected issue.

Next, participants answered the same four questions from Experiment 1 about their expectations of effective counter-arguing, which were averaged to form a composite measure ( $\alpha$ $=.95$ ). Finally, they were given a choice between counter-arguing (i.e., "Argue against the facts of the message on this page.") or engaging in untestability (i.e., "Argue that your position is ultimately a matter of opinion."), and completed the task of their choice.

\section{Results}

Like Experiment 1, we first ensured that participants were likely to be highly motivated to defend their beliefs. A one-sample t-test revealed that participant ratings of personal issue importance $(M=6.41, S D=1.83)$ were significantly above the midpoint (5) on the 9-point scale, $t(212)=11.23, p<.001,95 \%$ CI $[1.16,1.65]$

Did exposure to strong (vs. weak) belief-threatening information cause participants to avoid counter-arguing? Replicating Experiment 1, participants showed significantly stronger preferences for using untestability over counter-arguing when the message was strong than when 
it was weak, $(b=.50, S E=.28), z(211)=1.80, p=.036,95 \% \mathrm{CI}[.04, .96]$. That is, relative to receiving a weak message, receiving a strong message against their beliefs led to $65 \%$ greater odds of choosing untestability over counter-arguing, $\mathrm{OR}=1.65,95 \% \mathrm{CI}[1.04,2.61]$ (See Table 2).

Table 2.

Percentage of participants choosing each defense strategy as a function of condition

\begin{tabular}{ccc}
\hline & Weak condition & Strong condition \\
\cline { 2 - 3 } Defense Strategy & \multicolumn{2}{c}{ Percentage of participants } \\
\hline Counter-argue & .50 & .38 \\
Untestability & .50 & .62 \\
\hline
\end{tabular}

Was participants' preference for untestability over counter-arguing mediated by expectations of effective counter-arguing? Using the same mediation model as Experiment 1, results indicate yes. As expected, exposure to a strong message (as compared to a weak message) significantly lowered participants' expectations of effectively counter-arguing, $(b=-1.16, S E$ $=.28), t(211)=-4.13, p<.001,95 \% \mathrm{CI}[-1.72,-.61]$, which was associated with a significantly stronger preference for untestability, $(b=-.51, S E=.09), z(210)=5.48, p<.001,95 \%$ CI [-.69, -.32] (see Figure 2). Put another way, receiving a weak belief-threatening message produced higher expectations of effective counter-arguing, which was associated with a stronger preference for counter-arguing. Bootstrapping revealed that the indirect effect was significantly different from zero $\left(b=.59, S E_{\mathrm{boot}}=.18\right), 95 \% \mathrm{Cl}_{\mathrm{boot}}[.25,1.09]$. A test of moderated mediation revealed that the effects for the two issues were not significantly different from each other $(b$ $\left.=.26, S E_{\text {boot }}=.32\right), 95 \% \mathrm{Cl}_{\text {boot }}[-.40, .90]$.

Importantly, as in Experiment 1, expectations of effective counter-arguing was strongly associated with a preference for counter-arguing even with argument strength in the model, OR $=.60,95 \% \mathrm{CI}[.52, .70]$. That is, every unit increase in expectations of effective counter-arguing 
was associated with $40 \%$ lower odds of choosing untestibility (i.e., higher odds of choosing to counter-argue).

Figure 2. Mediation model testing the indirect effect of condition (weak vs. strong) on choice of defense strategy (counter-argue vs. untestability) through expectations of effective counterarguing.
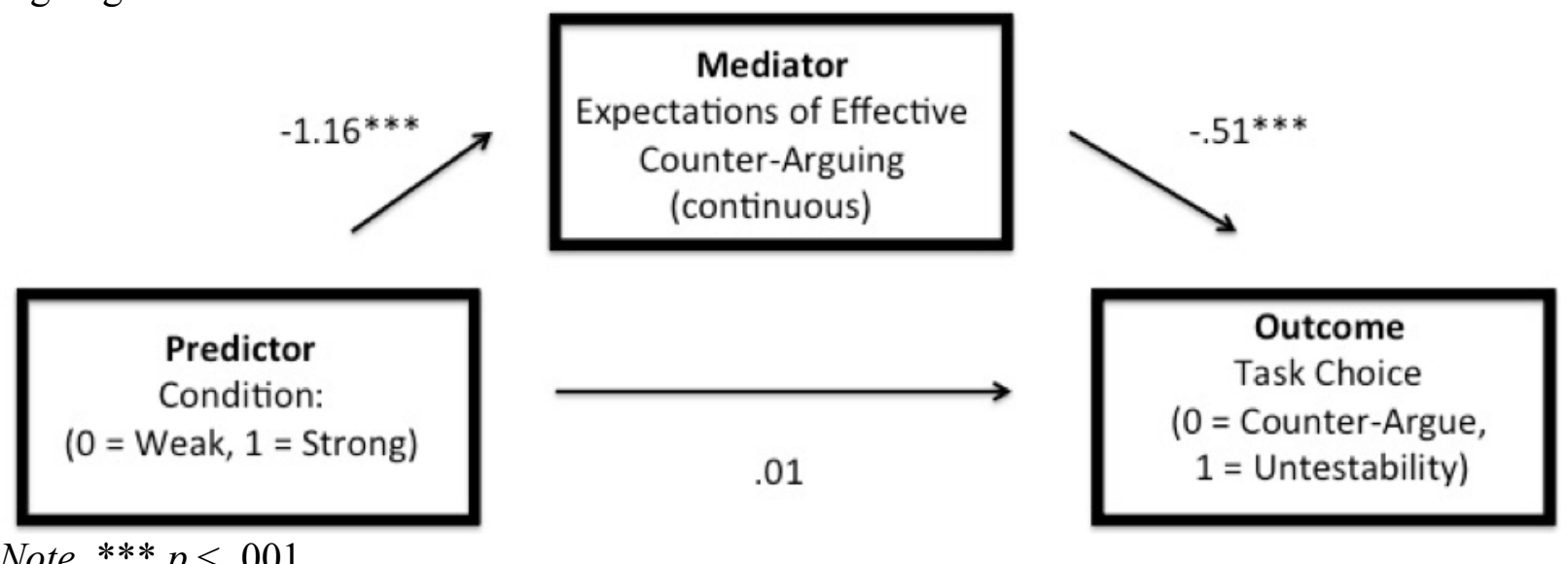

Note. $* * * p<.001$

As in Experiment 1, we also tested if issue importance moderated the path from expectations of effective counter-arguing to choice of belief-defense. A test of moderated mediation was not significant, $\left(b=.04, S E_{\text {boot }}=.07\right), 95 \% \mathrm{CI}_{\text {boot }}[-.12, .19]$.

\section{Meta-analysis}

We used the JASP statistical software to conduct random effects meta-analyses of both studies. Because these are the only two experiments we conducted testing this research question, this provides an unbiased meta-analytic effect size estimate. We did so for the path from condition (weak vs. strong) to expectations of effective counter-arguing, the path from expectations of effective counter-arguing to choice of defense strategy, as well as the indirect effect. All meta-analytic effects were significant. That is, compared to when participants were exposed to a weak message, exposure to a strong message led to significantly lower expectations of effective counter-arguing, $(b=-1.21), 95 \% \mathrm{CI}[-1.53,-.88]$. Further, expectations of effective counter-arguing was associated with decisions to counter-argue, $(b=-.50), 95 \% \mathrm{CI}[-.61,-.39]$. 
Together, these findings produced a significant meta-analytic indirect effect of argument strength condition on choice of defense strategy through expectations of effective counter-arguing, $(b$ $=.60), 95 \% \mathrm{CI}[.39, .82]$.

\section{Discussion}

Findings from Experiment 2 demonstrate that strong belief-inconsistent information caused people to believe that they cannot effectively counter-argue against a threat to their belief, which was associated with their preference for arguing that their position is a matter of opinion rather than a matter of fact. This is a non-trivial concession. It is as if participants concede, "I may not be able to prove that I'm right, but I cannot be proven wrong if it is a matter of opinion." This shows that people weaken their own position as a means of keeping it out of reach of empirical criticism. These findings support predictions derived from self-efficacy theory.

Counter-arguing appears to be a relatively more desirable way to defend one's beliefs as long as one can successfully do so-when self-efficacy is high. The evidence for this claim is especially strong in this experiment considering that high expectations of effective counterarguing was strongly associated with choosing to counter-argue, regardless of whether the argument was weak or strong. Consistent with self-efficacy theory, people highly motivated to fulfill the goal (belief-defense) will engage in the best possible strategy even if it requires more effort.

These results show that when counter-arguing is not possible, people move on to the epistemically weaker defense strategy of untestability so they will not be proven wrong and have to forfeit the belief altogether. This is consistent with research showing that people engage with belief-discrepant content when it is weak, but resort to reducing its impact when it is strong (Ahluwalia, 2000). However, the current experiment provides a theoretical advance by showing 
that self-efficacy predicts the method by which one pursues the goal of belief-defense, when multiple methods are available.

\section{General Discussion}

The research reported here is the first to examine people's choices of defense strategies in response to belief-threatening information. Two experiments demonstrated that people's preference to counter-argue is associated with their expectations that they will be successful, but expectations that they will be unsuccessful is associated people's preferences for alternative strategies. Importantly, we show that people do not simply choose the easiest method of beliefdefense, but rather what they believe will be most effective. Furthermore, we demonstrate that self-efficacy theory serves as a useful framework for predicting people's methods of beliefdefense.

These findings provide a notable theoretical advance by integrating previous work on cognitive dissonance and selective exposure into self-efficacy theory. For example, the current research refines the literature on selective exposure by suggesting that people do not broadly avoid or ignore belief-disconfirming information, but rather selectively engage with information they believe they can refute. Our analysis of mechanism shows that people's expectations for effective counter-arguing is an underlying factor in explaining whether people choose to engage with information that contradicts their beliefs, avoid it, or frame their position in untestable terms.

Importantly, the current findings and theoretical integration expand upon self-efficacy theory by investigating how people choose to pursue a goal when there are multiple paths for doing so. Going into the current research, it was unclear how people choose among methods of belief-defense. The results reported here, however, suggest that counter-arguing is a relatively 
more preferable method of belief-defense than ignoring or untestability. This fits with SET, which predicts that people will invest the necessary effort to attain a goal as long as self-efficacy is sufficiently high. Additionally, the current research shows that low self-efficacy is not associated with the abandonment of goal pursuit altogether, but rather people accept alternative methods of attaining the goal.

This theoretical framework for predicting people's methods of belief defense has crucial practical implications because it can inform applied research into messaging strategies that refute likely methods of belief defense before people can use them. For example, a cigarette smoker might respond to an anti-smoking advertisement by affirming that he is not taking a large health risk, considering his friends smoke more cigarettes than he does (e.g., defense via social comparison). This would suggest that a more effective messaging campaign would also address the amount one smokes. Applied research is needed to confirm this with direct empirical testing.

Recent research uses similar logic by debunking popular misinformation before communicating the scientific consensus on climate change (van der Linden, Leiserowitz, Rosenthal, \& Maibach, 2017). That is, many who reject that climate change is happening and is human-caused cite the "Petition Project" as evidence, which purports to have more than 31,000 signatures from American scientists claiming that climate change is not happening. van der Linden and colleagues (2017) found that messages about the true scientific consensus on climate change among climate scientists was significantly more effective when they preemptively debunked the fake petition (which had Charles Darwin and members of the Spice Girls as signatories). In our view, this method of science communication would be especially effective in other topical areas when scientists could accurately predict which method of belief defense people are likely to use. 
It would be fruitful to further expand the SET framework by testing when people prefer other defense strategies such as source derogation or appeals to consensus, for example. Consider an example in which people respond to belief-threatening scientific abstracts by arguing that science is not fit to address the issue in question (Munro, 2010). One's strong support for science would constrain one's defense strategies, making alternative strategies more attractive for keeping the belief intact. Thus, research on self-efficacy would benefit from further research into competing goals.

Additionally, future research should explore how results change when people are given more than two methods of belief-defense to choose from. In day-to-day arguments, it is likely people often have more than two options. Perhaps even better, future research should aim for a more ecologically valid approach where participants can respond without any constraints (e.g., open response), and their responses may be coded for their spontaneous methods of beliefdefense. Finally, participants in our experiments were not given the option to concede and change their views. Although it is unlikely participants will change their highly important beliefs from a simple experimental manipulation, it would be informative to learn what predicts concessions versus belief-defense.

One limitation of the current experiments is that we did not manipulate efficacy directly. Thus, to confirm a causal relationship between efficacy and choice of belief-defense, future research should experimentally manipulate perceived efficacy to determine its effects on choice of defense strategy. One potential way to do this is to inform participants of the task success rate of previous participants (e.g., 15\% vs. 85\%). Further, additional research of this sort should aim to identify additional mechanisms that may account for the relationship between efficacy and 
methods of belief-defense, such as expectations that one will become more certain of their existing attitudes after counter-arguing (e.g., Tormala \& Petty, 2002; 2004).

An additional limitation of the current experiments is that we only tested two issues. It is plausible that some issues are better fit for some defense strategies than others. For example, people might have a stronger moral opinion for their position on abortion or the death penalty than on taxes. For moral issues, then, it might not be as useful to argue using facts. This could help explain why merely labeling an attitude as moral makes it more resistant to persuasion (Luttrell, Petty, Briñol, \& Wagner, 2016)—because facts are not needed for a strong moral position. Thus, future research should address whether people's choice of defense strategy changes depending on how well the strategy fits the issue. Nonetheless, it is still encouraging that our findings were robust across two very different and important issues: gun control and the death penalty.

In conclusion, people have many methods of defending their beliefs from criticism, and choose which method to adopt in a given situation depending on how likely the method is to help them successfully defend those beliefs. While counter-arguing engages the belief-threatening information in a straightforward manner as a means to refute it, and is epistemically beneficial, task difficulty, such as encountering very strong arguments, may prevent people from using such a strategy. Thus, people resort to weaker strategies when counter-arguing is not possible as a means to avoid having to forfeit the belief altogether. Uncovering the strategies that people use, and their reasons for using them, has the potential to identify the barriers that stand in the way of communication campaigns and a more productive public discourse.

\section{Footnotes}

${ }^{1}$ Six participants were in the weak condition and four participants were in the strong condition. 


\footnotetext{
${ }^{2}$ All data and materials will remain on this repository for confirmation purposes for a minimum of 5 years. All measures, manipulations, and exclusions are disclosed. Sample size was decided before data collection of each study and did not continue after analysis.

${ }^{3}$ Other measures, related but not central to our hypotheses, were included for exploratory purposes including participant ideology, sources of political information (e.g., social media, television), and perceived knowledge and attitude certainty about issues in the experiment (gun control and the death penalty; see OSF project page for the full survey).

${ }^{4}$ Arguments were pilot tested as part of a larger set of experiments unrelated to the current research. In all cases, strong arguments were rated significantly stronger than weak arguments (see OSF project page).

${ }^{5}$ Issue did not moderate any effects and therefore we collapsed across issue in both experiments.

${ }^{6}$ One participant was removed from this calculation because they entered 1988 as their age.

${ }^{7}$ Nine participants were in the weak condition and two participants were in the strong condition.
}

\section{References}

Ahluwalia, R. (2000). Examination of psychological processes underlying resistance to persuasion. Journal of Consumer Research, 27(2), 217-232.

Albarracín, D., \& Mitchell, A. L. (2004). The role of defensive confidence in preference for proattitudinal information: How believing that one is strong can sometimes be a defensive weakness. Personality and Social Psychology Bulletin, 30(12), 1565-1584.

Bandura, A. (1977). Self-efficacy: toward a unifying theory of behavioral change. Psychological Review, 84(2), 191-215.

Bandura, A., Barbaranelli, C., Caprara, G. V., \& Pastorelli, C. (1996). Multifaceted impact of self-efficacy beliefs on academic functioning. Child Development, 67(3), 1206-1222.

Ditto, P. H., \& Lopez, D. F. (1992). Motivated skepticism: Use of differential decision criteria for preferred and nonpreferred conclusions. Journal of Personality and Social Psychology, 63, 568-584.

Eagly, A. H., \& Chaiken, S. (1993). The psychology of attitudes. Fort Worth, TX: Harcourt Brace Jovanovich.

Edwards, K., \& Smith, E. E. (1996). A disconfirmation bias in the evaluation of arguments. Journal of Personality and Social Psychology, 71(1), 5-24. 
Faul, F., Erdfelder, E., Lang, A. G., \& Buchner, A. (2007). G* Power 3: A flexible statistical power analysis program for the social, behavioral, and biomedical sciences. Behavior research methods, 39(2), 175-191.

Festinger, L. (1957). A Theory of Cognitive Dissonance, Stanford, CA: Stanford University Press.

Friesen, J. P., Campbell, T. H., \& Kay, A. C. (2015). The psychological advantage of unfalsifiability: The appeal of untestable religious and political ideologies. Journal of Personality and Social Psychology, 108(3), 515-530.

Goldberg, M. H. \& Carmichael, C. L. (2017). Language complexity, belief-consistency, and the evaluation of policies. Comprehensive Results in Social Psychology. 1-17.

Hart, W., Albarracín, D., Eagly, A. H., Brechan, I., Lindberg, M. J., \& Merrill, L. (2009). Feeling validated versus being correct: a meta-analysis of selective exposure to information. Psychological Bulletin, 135, 555-588.

Hayes, A. F. (2013). Introduction to mediation, moderation, and conditional process analysis: A regression-based approach. New York: Guilford Press.

Jost, J. T., Banaji, M. R., \& Nosek, B. A. (2004). A decade of system justification theory: Accumulated evidence of conscious and unconscious bolstering of the status quo. Political Psychology, 25, 881-919.

Kruglanski, A. W. (1996). Motivated social cognition: Principles of the interface. In E. T. Higgins \& A. W. Kruglanski (Eds.), Social psychology: Handbook of basic principles (pp. 493-520). New York, NY: Guilford Press.

Kunda, Z. (1990). The case for motivated reasoning. Psychological Bulletin, 108, 480-498. 
Lowin, A. (1967). Approach and avoidance: alternate modes of selective exposure to information. Journal of Personality and Social Psychology, 6(1), 1-9.

Luttrell, A., Petty, R. E., Briñol, P., \& Wagner, B. C. (2016). Making it moral: Merely labeling an attitude as moral increases its strength. Journal of Experimental Social Psychology, $65,82-93$.

Munro, G.D.(2010) The scientific impotence excuse: discounting belief-threatening scientific abstracts. Journal of Applied Social Psychology, 40, 3, 579-600.

Rios, K., Goldberg, M. H., \& Totton, R. R. (2018). An Informational Influence Perspective on (Non) conformity: Perceived Knowledgeability Increases Expression of Minority Opinions. Communication Research. 45(2), 241-260.

Sedikides, C. (2010). Why does religiosity persist? Personality and Social Psychology Review, $14,3-6$.

Stajkovic, A. D., \& Luthans, F. (1998). Self-efficacy and work-related performance: A metaanalysis. Psychological Bulletin, 124(2), 240-261.

Swann Jr, W. B. (2011). Self-verification theory. Handbook of theories of social psychology, 2, $23-42$.

Tormala, Z. L., \& Petty, R. E. (2002). What doesn't kill me makes me stronger: The effects of resisting persuasion on attitude certainty. Journal of personality and social psychology, 83(6), 1298-1313.

Tormala, Z. L., \& Petty, R. E. (2004). Source credibility and attitude certainty: A metacognitive analysis of resistance to persuasion. Journal of Consumer Psychology, 14(4), 427-442.

van der Linden, S., Leiserowitz, A., Rosenthal, S., \& Maibach, E. (2017). Inoculating the public against misinformation about climate change. Global Challenges, 1(2), 1600008. 
Zhou, H., \& Fishbach, A. (2016). The pitfall of experimenting on the web: How unattended selective attrition leads to surprising (yet false) research conclusions. Journal of Personality and Social Psychology, 111(4), 493-504. 